\title{
Hetero-Diels-Alder reactions of $N$-phosphoryltrihaloacetimidoyl chlorides with 1,3-butadienes
}

\author{
Mykola V. Kolotylo, Oleksii A. Synytsya, and Petro P. Onys'ko* \\ Institute of Organic Chemistry, National Academy of Sciences, \\ 5 Murmans 'ka St, 02660, Kyiv, Ukraine \\ E-mail:onysko@rambler.ru
}

\section{Dedicated to Prof. Pawel Kafarski to honor the achievements within his career}

\begin{abstract}
Hetero-Diels-Alder reactions of $N$-phosphoryltrihaloacetimidoyl chlorides with 1,3-butadienes led to new 2-chloro-2-trihalomethyl substituted tetrahydropyridine adducts. Cycloadducts with $\mathrm{CF}_{3}$ group undergo thermal or acid catalyzed dehydrochlorination affording respective dihydropyridines and subsequent aromatization to form 2-trifluoromethylpyridines. Trichloromethyl analogs under similar conditions undergo aromatization accompanied by unusual reduction of $\mathrm{CCl}_{3}$ group to form respective 2-dichloromethyl substituted pyridines.
\end{abstract}

Keywords: Aza-Diels-Alder reaction, imidoyl chlorides, tetrahydropyridines, dihydropyridines, pyridines

\section{Introduction}

Imidoyl chlorides combine the properties of acid chlorides and azomethynes. They are reactive and versatile chemical agents and have found wide application in organic synthesis and in the study of chemical reactivity. ${ }^{1}$ Trihaloacetimidoyl chlorides are regarded as new promising building blocks for regioselective introduction of (poly)haloalkyl groups into acyclic or heterocyclic compounds. ${ }^{2}$ Cycloaddition across $\mathrm{C}=\mathrm{N}$ bond of trihaloacetimidoyl chlorides would offer additional opportunities connected with the possibility to introduce regioselectively trihalomethyl group into a molecule and further transformations of primary products via chlorine atom participation. Replacement of hydrocarbon groups in molecules with their fluorinated analogs imparts a variety of useful properties to certain medicines, including enhanced binding interactions and metabolic stability. ${ }^{3}$ As a result, fluorine containing compounds are becoming increasingly important in both agrochemistry and medicine. ${ }^{4}$ On the other hand polychloroalkyl derivatives reveals as a rule greater herbicidal and fungicidal activity then their fluorinated 
counterparts. ${ }^{5}$ In the pyridine and dihydropyridine series a number of compounds bearing polyfluoro- and/or polyclorohaloalkyl substituents was found to possess useful pharmacological properties. ${ }^{6}$ At the same time the use of imidoyl chlorides for construction of heterocyclic ring by means of hetero-Diels-Alder reactions with 1,3-dienes remains almost unexplored. ${ }^{7}$

Now we report on a novel approach for hydrogenated $\alpha$-polyhalomethyl containing pyridines and respective pyridines based on cycloaddition reactions of $\mathrm{N}$ phosphoryltrihaloacetimidoyl chlorides with 1,3-butadienes.

\section{Results and Discussion}

We have found that $N$-dichlorophosphoryltrihaloacetimidoyl chlorides $\mathbf{1 a , b}$ readily react with 1,3-dienes $\mathbf{2 a , b}$ under mild conditions to afford functionalized tetrahydropyridines 3a-d (Scheme 1). Remarkably, the reaction with non-symmetrical diene $\mathbf{2 b}$ proceeds regioselectively with the formation of 4-methyl substituted isomers $\mathbf{3 b}, \mathbf{d}$.

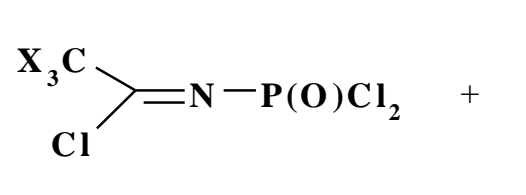

$\mathbf{1 a , b}$

$1 \mathbf{a} \mathbf{X}=\mathbf{F}$

1b $\mathrm{X}=\mathrm{Cl}$

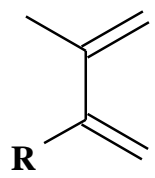

$\mathbf{2 a}, \mathbf{b}$

2a $\mathbf{R}=\mathbf{M e}$

2b $\mathbf{R}=\mathbf{H}$

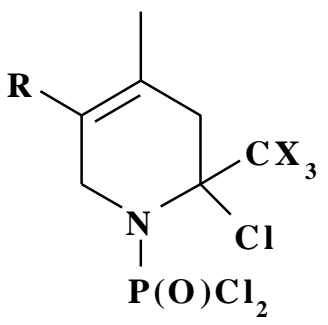

3a-d

3a $X=F, R=M e$

3 b $X=F, R=H$

3c $\mathrm{X}=\mathrm{Cl}, \mathrm{R}=\mathrm{Me}$

3d $\mathbf{X}=\mathbf{C l}, \mathbf{R}=\mathbf{H}$

\section{Scheme 1}

Cycloaddition is favored by electron-withdrawing substituents $\mathrm{X}$ in imine $\mathbf{1}\left(\mathrm{CF}_{3}>\mathrm{CCl}_{3}\right)$ and electron releasing substituents $\mathrm{R}$ in diene $\mathbf{2}(\mathrm{Me}>\mathrm{H})$. Thus, reaction of $\mathbf{1 a}$ with $\mathbf{2}$ easily proceeds at room temperature and with exothermic effect (in case of $\mathbf{2 a}$ ), while trichloro analog $\mathbf{1 b}$ reacts with $\mathbf{2 a}$ only at heating (ether, $5 \mathrm{hrs}$ ). For the completion of reaction of $\mathbf{1 b}$ and $\mathbf{2 b}$ boiling in benzene is necessary. Under these conditions in addition to 3d, 50\%, 5-chloro-6dichloromethyl-1-dichlorophosphoryl-4-methyl-1,2-dihydropyridine 4, 12\%, and 2-chloro-4methyl-2-trichloromethyl-1,2,3,6-tetrahydropyridine 5, 3\%, were isolated by column chromatography. Replacing the dichlorophosphoryl group at nitrogen atom in 1a with less electron-withdrawing $(\mathrm{EtO})_{2} \mathrm{P}(\mathrm{O})$ substituent $\left(\sigma_{\mathrm{I}} 0.65\right.$ and 0.35 , respectively), ${ }^{8}$ essentially retards its reactivity toward 2 . The reaction of respective imidoyl chloride, $\mathrm{CF}_{3} \mathrm{C}(\mathrm{Cl})=\mathrm{NP}(\mathrm{O})(\mathrm{OEt})_{2}$, with 
diene 2a proceeds only at prolonged heating in benzene; cycloaddition under these conditions is accompanied by other processes leading to a complex mixture. As a result, cycloaddition product 6 was not isolated and detected only spectrally $\left(\delta_{\mathrm{F}}-63.7 \mathrm{ppm}, \delta_{\mathrm{P}} 8.3 \mathrm{ppm}\right)$.

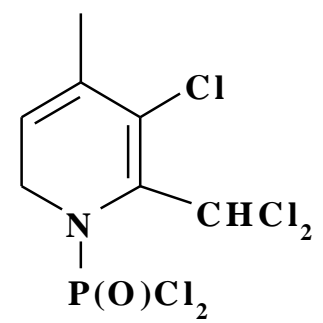

4

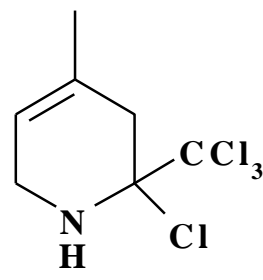

5

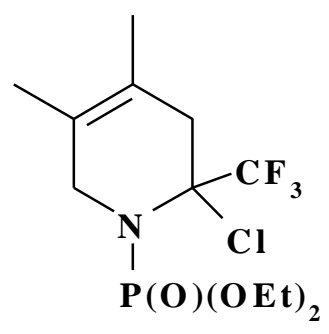

6

Tetrahydropyridines 3c,d bearing trichloromethyl group are relatively stable crystalline compounds and can be kept at room temperature for several months. At the same time their trifluoro analogs 3a,b upon distillation in vacuum eliminate hydrogen chloride to afford dihydropyridines 7a,b (Scheme 2).

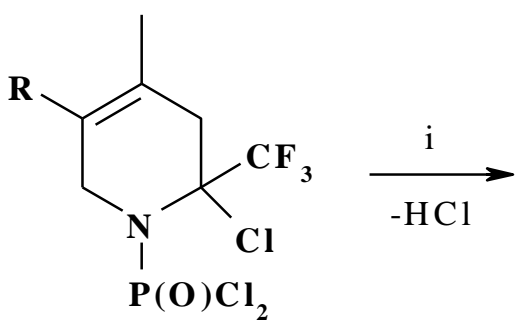

3a,b

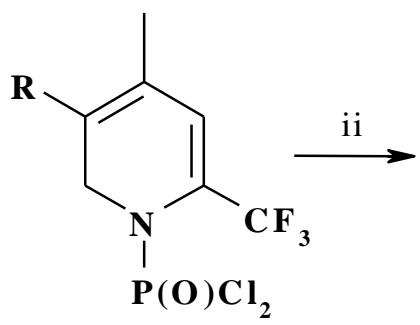

7a,b<smiles>[R]c1cnc(C(F)(F)F)cc1C</smiles>

$8 a, b$

Scheme 2. Reagents and conditions: i) $\Delta$ or $\mathrm{CF}_{3} \mathrm{COOD}$; ii) aq $\mathrm{HCl}$-dioxane or $\Delta$.

Slow transformation $\mathbf{3 a} \rightarrow \mathbf{7 a}$ occurs even at room temperature. Nitrogen bases (DBU, $\mathrm{DABCO}, \mathrm{Et}_{3} \mathrm{~N}$ ) or carboxylic acid additives favor the reaction. Thus, in solutions of $\mathrm{CF}_{3} \mathrm{COOD}$ the elimination of $\mathrm{HCl}$ from 3a,b is completed within $24 \mathrm{hrs}$ at room temperature. The same is true for $\mathrm{CF}_{3} \mathrm{COOH}$ or $\mathrm{AcOH}$ solutions but in these cases the formation of $\mathbf{7}$ is complicated by their partial transformation into respective pyridines 8. Dihydropyridines 7 are oily rather unstable compounds, easily soluble in organic solvents. Even at room temperatures they are gradually ( 4 weeks) converted into resinous-like hardly soluble substances. Upon thermal distillation or heating in aq $\mathrm{HCl}$-dioxane compounds 7 undergo aromatization to afford 2trifluoromethyl substituted pyridines $\mathbf{8}$ in $63-81 \%$ yields.

The behavior of 2-trichloromethyl tetrahydropyridines 3c,d is quite different from that of their trifluoromethyl analogs. Upon distillation in vacuum, heating in aq $\mathrm{HCl}$-dioxane solution, 
or on treatment with $\mathrm{CF}_{3} \mathrm{COOH}$, they give 2-dichloromethyl pyridines 9 (Scheme 3) rather than expected pyridines with trichloromethyl group.

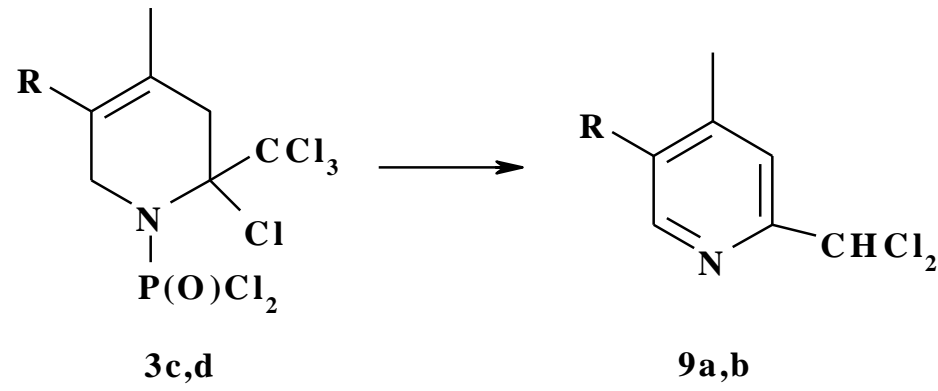

\section{Scheme 3}

Reduction of trichloromethyl group during conversion of tetrahydropyridines $\mathbf{3 c}, \mathbf{d}$ to respective pyridines $\mathbf{9 a , b}$ is quite unexpected. The following peculiarities are characteristic of this unusual transformation: 1) the reaction is promoted by acids; 2) the intermediate dihydropyridines analogous to 7 (Scheme 2) were not detected in the course reaction; 3) the performance of the reaction in $\mathrm{CF}_{3} \mathrm{COOD}$ does not lead to incorporation of the of deuterium into dichloromethyl group of $\mathbf{9}$. Hydrophosphoryl compounds were reported to convert activated trichloromethyl group into dichloromethyl one. ${ }^{9}$ In view of these data we first regarded the possibility of reduction of $\mathrm{CCl}_{3}$ group with the participation of $\mathrm{Cl}_{2} \mathrm{POH}$, that could be in principle generated from 3c,d. However, our attempts to execute reduction of trichloromethyl group of model 4,5-dimethyl-2-(trichloromethyl)pyridine ${ }^{10}$ by reacting it with diethylphosphonate, $(\mathrm{EtO})_{2} \mathrm{P}(\mathrm{O}) \mathrm{H}$, under different conditions, failed.

Taking into account the above observations the following pathway of reaction should be outlined (Scheme 4).

Generation of positive charge at phosphorus atom in intermediate $A$ promotes the rupture of $\mathrm{P}-\mathrm{N}$ bond and facilitates the elimination of $\mathrm{POCl}_{3}$. Release of sterical strains caused by voluminous 1,2-substituents in $A$ also favours transformation $A \rightarrow B$. The greater sterical volume of trichloromethyl group as compared with trifluoromethyl one is likely one of the reasons accounting for distinctions in behavior of tetrahydropyridines $\mathbf{3 a , b}$ and $\mathbf{3 c , d}$. Electronwithdrawing $\mathrm{CCl}_{3}$ group and positive charge on nitrogen atom obviously promotes 1,3-proton shift $(B \rightarrow C)$ in $\mathrm{C}-\mathrm{N}=\mathrm{C}$ triad; ${ }^{2 \mathrm{c}, 11}$ moreover, in azaallylic triad proton tends to migrate to the carbon atom bearing more electron-withdrawing substituent. ${ }^{11,12}$ Finally, elimination of hydrogen chloride in $C$, favored by the presence of onium center, and subsequent aromatization of intermediate $D$ by means of prototropic shift affords dichloromethylpyridines 9 . It is worthwhile to note that according to proposed scheme hydrogen of $\mathrm{CHCl}_{2}$ groups comes from hydrogenated pyridine ring rather than from outer source, accounting for the absence of $\mathrm{H}-\mathrm{D}$ exchange in the cause of $\mathrm{CCl}_{3} \rightarrow \mathrm{CHCl}_{2}$ conversion. 


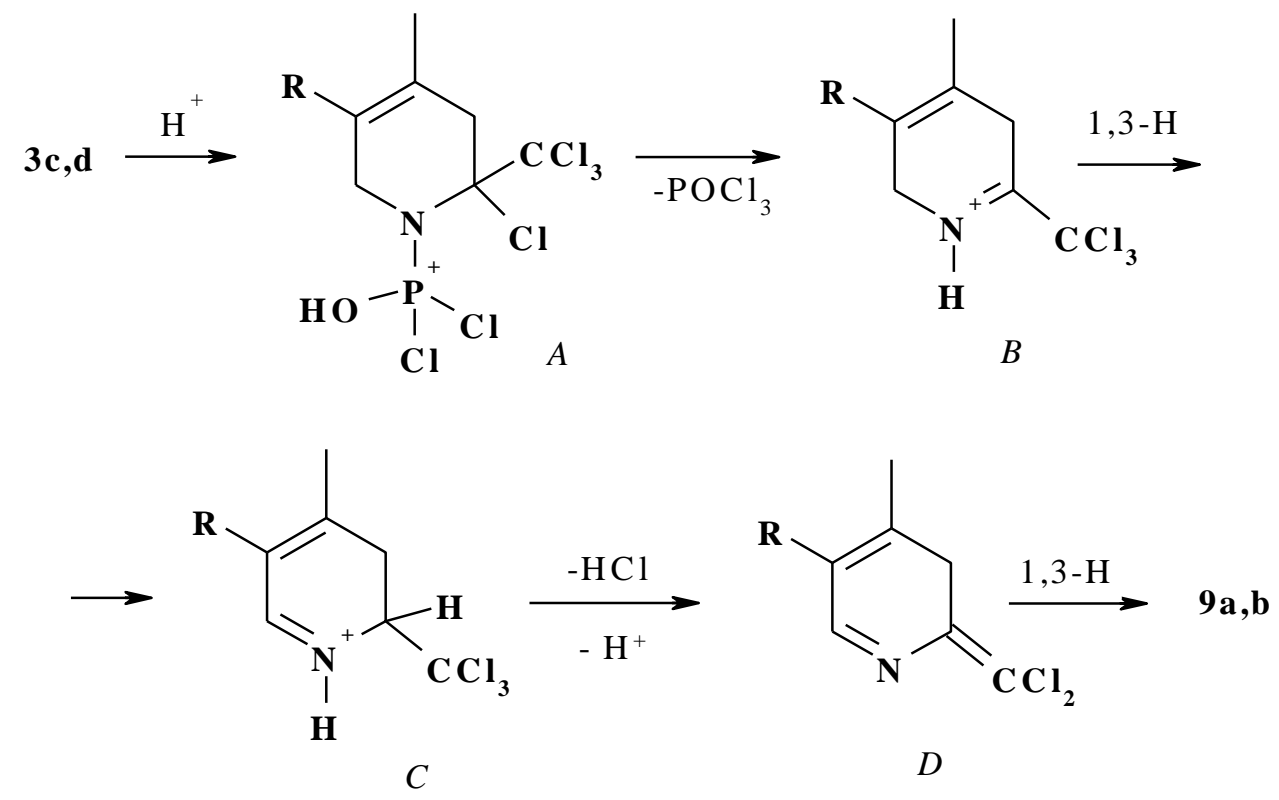

\section{Scheme 4}

The spectral and analytical data of compounds obtained are in complete agreement with their structure. The hydrogenated trifluoromethylpyridines $\mathbf{3 a}, \mathbf{b}, \mathbf{7 a}, \mathbf{b}$, and pyridines $\mathbf{8 a}, \mathbf{b}$ give clearly distinguishable ${ }^{19} \mathrm{~F}$ NMR characteristics $\left(\delta_{\mathrm{F}}-75.8 \div-77.4 \mathrm{ppm},-63.2 \div-63.3 \mathrm{ppm}\right.$, and $-67.4 \div-68.8 \mathrm{ppm}$, respectively) that allow easy monitoring of the process and identification of the products in the reaction mixture. The regiochemistry of cycloaddition with non-symmetrical diene $\mathbf{2 b}$ was unambiguously confirmed by ${ }^{1} \mathrm{H}$ NMR spectra of pyridines $\mathbf{8 b}, \mathbf{9 b}$ in which characteristic splitting of the 6- $\mathrm{H}$ signals was observed: $\delta 8.4-8.6 \mathrm{ppm},{ }^{3} \mathrm{~J}_{\mathrm{H}_{6} \mathrm{H}_{5}} 5 \mathrm{~Hz}$.

\section{Conclusions}

In summary, based on hetero Diels-Alder reactions of $N$-phosphoryltrihaloacetimidoyl chlorides with 1,3-butadienes, we have developed a simple and efficient synthesis of functionalized 2trihalomethyl tetrahydropyridines, 2-trifluoromethyl dihydropyridines, and pyridines bearing $\mathrm{CHCl}_{2}$ or $\mathrm{CF}_{3}$ group in $\alpha$-position.

\section{Experimental Section}

General. IR spectra were obtained with an UR-20 instrument. ${ }^{1} \mathrm{H},{ }^{13} \mathrm{C}$ NMR spectra were recorded on Bruker Avance DRX 500 instrument operating at 500.07 and $125.76 \mathrm{MHz}$, respectively, ${ }^{19} \mathrm{~F}$ NMR and ${ }^{31} \mathrm{P}$ NMR spectra - on Gemini 200 Varian instrument operating at 188.14 and $80.95 \mathrm{MHz}$ respectively. Chemical shifts are reported relative to TMS $\left({ }^{1} \mathrm{H},{ }^{13} \mathrm{C}\right)$, and 
$\mathrm{CFCl}_{3}\left({ }^{19} \mathrm{~F}\right)$ as the internal standards or relative to external $85 \% \mathrm{H}_{3} \mathrm{PO}_{4}\left({ }^{31} \mathrm{P}\right)$. APCI MS spectra were recorded using Agilent 1100 instrument. Melting points are uncorrected. Solvents were dried before use according to standard methods. All reactions were carried out under argon atmosphere.

2-Chloro-1-dichlorophosphoryl-4,5-dimethyl-2-(trifluoromethyl)-1,2,3,6-tetrahydro-pyridine (3a). A solution of the 2,3-dimethylbutadiene $2 \mathbf{a}(1.2 \mathrm{~g}, 15 \mathrm{mmol})$ in $5 \mathrm{~mL}$ of diethyl ether was added to a stirred solution of imidoyl chloride $\mathbf{1 a}^{13}(2.48 \mathrm{~g}, 10 \mathrm{mmol})$ in $10 \mathrm{~mL}$ of diethyl ether. After stirring at room temperature for $5 \mathrm{hrs}$ the reaction mixture was evaporated in vacuum at $40-50{ }^{\circ} \mathrm{C}$ to afford compound 3a. Yield: $2.67 \mathrm{~g}(81 \%)$, white solid; mp $81-83^{\circ} \mathrm{C}$ (hexane). ${ }^{1} \mathrm{H}$ $\operatorname{NMR}\left(\mathrm{CDCl}_{3}\right): \delta=1.88\left(\mathrm{~s}, 6 \mathrm{H}, 4,5-\mathrm{Me}_{2}\right), 2.82\left(\mathrm{~m}, 2 \mathrm{H}, \mathrm{CCH}_{2}\right), 3.68\left(\mathrm{dd},{ }^{2} J_{\mathrm{HH}}=13 \mathrm{~Hz},{ }^{3} J_{\mathrm{HP}}=14\right.$ $\left.\mathrm{Hz}, 1 \mathrm{H}, \mathrm{NCH}_{\mathrm{A}}\right), 3.92\left(\mathrm{dd},{ }^{2} J_{\mathrm{HH}}=13 \mathrm{~Hz},{ }^{3} J_{\mathrm{HP}}=15 \mathrm{~Hz}, 1 \mathrm{H}, \mathrm{NCH}_{\mathrm{B}}\right) .{ }^{13} \mathrm{C} \mathrm{NMR}\left(\mathrm{CDCl}_{3}\right): \delta=$ 17.05, 18.60 (4-Me, 5-Me), $42.29\left(\mathrm{~d},{ }^{3} J_{\mathrm{CP}}=6 \mathrm{~Hz}, 3-\mathrm{C}\right), 50.77\left(\mathrm{~d},{ }^{2} J_{\mathrm{CP}}=4 \mathrm{~Hz}, 6-\mathrm{C}\right), 81.78$ (q, $\left.{ }^{2} J_{\mathrm{CF}}=33 \mathrm{~Hz}, 2-\mathrm{C}\right), 122.50\left(\mathrm{q},{ }^{1} J_{\mathrm{CF}}=285 \mathrm{~Hz}, \mathrm{CF}_{3}\right) 126.62(4-\mathrm{C}), 126.75\left(\mathrm{~d}, J_{\mathrm{CP}}=3 \mathrm{~Hz}\right)(5-\mathrm{C}) .{ }^{19} \mathrm{~F}$ $\operatorname{NMR}\left(\mathrm{CDCl}_{3}\right): \delta=-75.75 \mathrm{~s} .{ }^{31} \mathrm{P} \operatorname{NMR}\left(\mathrm{CDCl}_{3}\right): \delta=14.0\left(\mathrm{dd},{ }^{3} J_{\mathrm{PH}_{\mathrm{A}}}=13 \mathrm{~Hz},{ }^{3} J_{\mathrm{PH}_{\mathrm{B}}}=15 \mathrm{~Hz}\right)$. Anal. Calcd for $\mathrm{C}_{8} \mathrm{H}_{10} \mathrm{Cl}_{3} \mathrm{~F}_{3} \mathrm{NOP}$ : C 29.07; $\mathrm{H}$ 3.05; $\mathrm{Cl} 32.18 ; \mathrm{P} 9.37$. Found: $\mathrm{C} 28.89 ; \mathrm{H} \mathrm{3.09;} \mathrm{Cl}$ 31.87; P 9.32.

\section{2-Chloro-1-dichlorophosphoryl-4-methyl-2-(trifluoromethyl)-1,2,3,6-tetrahydropyridine}

(3b) was synthesized analogously to 3a by stirring ethereal solution of imidoyl chloride 1a (2.48 g, $10 \mathrm{mmol})$ and 2-methylbutadiene $2 \mathbf{b}(1.02 \mathrm{~g}, 15 \mathrm{mmol})$ for $25 \mathrm{hrs}$ at room temperature. Compound 3b was purified by column chromatography (silica gel, hexane/ethyl acetate 10:1). Yield: $2.2 \mathrm{~g}(70 \%)$, yellow oil. ${ }^{1} \mathrm{H} \mathrm{NMR}\left(\mathrm{CDCl}_{3}\right): \delta=1.91(\mathrm{~s}, 3 \mathrm{H}, \mathrm{Me}), 2.85\left(\mathrm{~m}, 2 \mathrm{H}, \mathrm{CCH}_{2}\right)$, $3.78\left(\mathrm{~m}, 1 \mathrm{H}, \mathrm{NCH}_{\mathrm{A}}\right), 4.06\left(\mathrm{~m}, 1 \mathrm{H}, \mathrm{NCH}_{\mathrm{B}}\right), 5.89(\mathrm{br}, 1 \mathrm{H}, 5-\mathrm{H}) .{ }^{13} \mathrm{C} \mathrm{NMR}\left(\mathrm{CDCl}_{3}\right): \delta=22.32(\mathrm{Me})$, $41.49\left(\mathrm{~d},{ }^{3} J_{\mathrm{CP}}=4 \mathrm{~Hz}, 3-\mathrm{C}\right), 46.18\left(\mathrm{~d},{ }^{2} J_{\mathrm{CP}}=5 \mathrm{~Hz}, 6-\mathrm{C}\right), 81.48\left(\mathrm{q},{ }^{2} J_{\mathrm{CF}}=35 \mathrm{~Hz}, 2-\mathrm{C}\right), 118.73(\mathrm{~d}$, $\left.{ }^{3} J_{\mathrm{CP}}=5 \mathrm{~Hz}, 5-\mathrm{C}\right), 122.50\left(\mathrm{q},{ }^{1} J_{\mathrm{CF}}=284 \mathrm{~Hz}, \mathrm{CF}_{3}\right), 134.66(4-\mathrm{C}) .{ }^{19} \mathrm{~F}$ NMR $\left(\mathrm{CDCl}_{3}\right): \delta=-77.42$. ${ }^{31} \mathrm{P} \mathrm{NMR}\left(\mathrm{CDCl}_{3}\right): \delta=10.9$. Anal. Calcd for $\mathrm{C}_{7} \mathrm{H}_{8} \mathrm{Cl}_{3} \mathrm{~F}_{3} \mathrm{NOP}: \mathrm{C} 26.57, \mathrm{H} 2.55, \mathrm{Cl} 33,61, \mathrm{~N} 4.43$, P 9.79. Found: C 26.91; H 2.43; Cl 33,11; N 4.68; P 9.85.

\section{2-Chloro-1-dichlorophosphoryl-4,5-dimethyl-2-(trichloromethyl)-1,2,3,6-tetrahydro-pyri-}

dine (3c) was synthesized analogously to $\mathbf{3 a}$ by refluxing ethereal solution of imidoyl chloride $\mathbf{1 b}^{14}(2.98 \mathrm{~g}, 10 \mathrm{mmol})$ and dimethylbutadiene $\mathbf{2 a}(1.2 \mathrm{~g}, 15 \mathrm{mmol})$ for $5 \mathrm{hrs}$. Yield: $2.95 \mathrm{~g}$ (78\%), white solid, mp 158-160 ${ }^{\circ} \mathrm{C}$ (hexane-benzene, 3:1). ${ }^{1} \mathrm{H}$ NMR $\left(\mathrm{CDCl}_{3}\right): \delta=1.92$ (s, 3H, $\mathrm{Me}), 1.94(\mathrm{~s}, 3 \mathrm{H}, \mathrm{Me}), 3.21\left(\mathrm{~m}, 2 \mathrm{H}, \mathrm{CCH}_{2}\right), 3.83\left(\mathrm{dd},{ }^{2} J_{\mathrm{HH}} \sim 14 \mathrm{~Hz},{ }^{3} J_{\mathrm{HP}} \sim 15 \mathrm{~Hz}, 1 \mathrm{H}, \mathrm{NCH}_{\mathrm{A}}\right)$, $4.03\left(\mathrm{dd},{ }^{2} J_{\mathrm{HH}}=14 \mathrm{~Hz},{ }^{3} J_{\mathrm{HP}} \sim 15 \mathrm{~Hz}, 1 \mathrm{H}, \mathrm{NCH}_{\mathrm{B}}\right) .{ }^{13} \mathrm{C} \mathrm{NMR}\left(\mathrm{CDCl}_{3}\right): \delta=17.22,18.24$ (4-Me, 5-Me), $45.59\left(\mathrm{~d},{ }^{3} J_{\mathrm{CP}}=5 \mathrm{~Hz}, 3-\mathrm{C}\right), 53.43\left(\mathrm{~d},{ }^{2} J_{\mathrm{CP}}=5 \mathrm{~Hz}, 6-\mathrm{C}\right), 93.82,105.86\left(2-\mathrm{C}, \mathrm{CCl}_{3}\right) 128.36$, 128.44 (4-C, 5-C). ${ }^{31} \mathrm{P}$ NMR $\left(\mathrm{CDCl}_{3}\right): \delta=13.26\left(\mathrm{t},{ }^{3} J_{\mathrm{PH}} \sim 15 \mathrm{~Hz}\right)$. Anal. Calcd for $\mathrm{C}_{8} \mathrm{H}_{10} \mathrm{Cl}_{6} \mathrm{NOP}$ : C 25.30; H 2.65; Cl 56.00; N 3.69; P 8.15. Found: C 24.98; H 2.91; Cl 55.87; N 3.57; P 7.87.

Compounds 3d, 4 and $\mathbf{5}$ were synthesized analogously to 3a by refluxing benzene solution of imidoyl chloride $\mathbf{1 b}(2.98 \mathrm{~g}, 10 \mathrm{mmol})$ and methylbutadiene $\mathbf{2 b}(1.02 \mathrm{~g}, 15 \mathrm{mmol})$ for $3 \mathrm{hrs}$. Column chromatography (silica gel, hexane/ethyl acetate 10:1) afforded tetrahydropyridines 3d, 5 and dihydropyridine 4. 
2-Chloro-1-dichlorophosphoryl-4-methyl-2-(trichloromethyl)tetrahydropyridine (3d). Yield $1.83 \mathrm{~g}(50 \%)$, yellow oil. ${ }^{1} \mathrm{H} \mathrm{NMR}\left(\mathrm{CDCl}_{3}\right): \delta=2.00(\mathrm{~s}, 3 \mathrm{H}, \mathrm{Me}), 3.26\left(\mathrm{~m}, 2 \mathrm{H}, \mathrm{CCH}_{2}\right), 3.74$ $\left(\mathrm{m}, 1 \mathrm{H}, \mathrm{NCH}_{\mathrm{A}}\right), 4.25\left(\mathrm{~m}, 1 \mathrm{H}, \mathrm{NCH}_{\mathrm{B}}\right), 6.11(\mathrm{~m}, 1 \mathrm{H}, 5-\mathrm{H}) .{ }^{13} \mathrm{C} \mathrm{NMR}\left(\mathrm{CDCl}_{3}\right): \delta=22.57(\mathrm{Me})$, $44.56\left(\mathrm{~d},{ }^{3} J_{\mathrm{CP}}=5 \mathrm{~Hz}, 3-\mathrm{C}\right), 48.51\left(\mathrm{~d},{ }^{2} J_{\mathrm{CP}}=6 \mathrm{~Hz}, 6-\mathrm{C}\right), 92.57,105.90\left(2-\mathrm{C}, \mathrm{CCl}_{3}\right), 121.00(5-\mathrm{C})$, $137.68(4-\mathrm{C}) .{ }^{31} \mathrm{P}$ NMR $\left(\mathrm{CDCl}_{3}\right): \delta=13.4\left(\mathrm{t},{ }^{3} \mathrm{~J}_{\mathrm{PH}}=16 \mathrm{~Hz}\right)$. Anal. Calcd for $\mathrm{C}_{7} \mathrm{H}_{8} \mathrm{Cl}{ }_{6} \mathrm{NOP}: \mathrm{C}$ 22.98; H, 2.20; Cl 58.15; N, 3.83; P, 8.47. Found: C 22,73; H 2.14; Cl 57,76; N 3.51; P 8.36.

5-Chloro-6-(dichloromethyl)-1-dichlorophosphoryl-4-methyl-1,2-dihydropyridine (4). Yield $0.35 \mathrm{~g}(12 \%)$, white solid, mp 129-131 ${ }^{\circ} \mathrm{C} .{ }^{1} \mathrm{H}$ NMR $\left(\mathrm{CDCl}_{3}\right): \delta=1.92(\mathrm{~s}, 3 \mathrm{H}, \mathrm{Me}), 3.85(\mathrm{~m}, 1 \mathrm{H}$, $\left.\mathrm{NCH}_{\mathrm{A}}\right), 4.18\left(\mathrm{~m}, 1 \mathrm{H}, \mathrm{NCH}_{\mathrm{B}}\right), 5.18(\mathrm{br}, 1 \mathrm{H}, 5-\mathrm{H}), 6.66\left(\mathrm{~s}, 1 \mathrm{H}, \mathrm{CHCl}_{2}\right) .{ }^{13} \mathrm{C} \mathrm{NMR}\left(\mathrm{CDCl}_{3}\right): \delta=$ $20.48(\mathrm{Me}), 46.33\left(\mathrm{~d},{ }^{2} J_{\mathrm{CP}}=7 \mathrm{~Hz}, 2-\mathrm{C}\right), 54.20\left(\mathrm{CHCl}_{2}\right), 120.59\left(\mathrm{~d},{ }^{3} J_{\mathrm{CP}}=7 \mathrm{~Hz}, 3-\mathrm{C}\right), 126.06(\mathrm{~d}$, $\left.{ }^{3} J_{\mathrm{CP}}=9 \mathrm{~Hz}, 5-\mathrm{C}\right), 130.92(4-\mathrm{C}), 133.63\left(\mathrm{~d},{ }^{2} J_{\mathrm{CP}}=1 \mathrm{~Hz}, 6-\mathrm{C}\right) .{ }^{31} \mathrm{P} \mathrm{NMR}\left(\mathrm{CDCl}_{3}\right): \delta=9.2\left(\mathrm{t},{ }^{3} J_{\mathrm{PH}}\right.$ $=12 \mathrm{~Hz}$ ). Anal. Calcd for $\mathrm{C}_{7} \mathrm{H}_{7} \mathrm{Cl}_{5} \mathrm{NOP}: \mathrm{C}, 25.53 ; \mathrm{H}, 2.14 ; \mathrm{Cl} 53.82 ; \mathrm{N}$ 4.25; P 9.40. Found: C 25.37; H 2.09; $\mathrm{Cl}$ 53.27; $\mathrm{N} 4.21 ; \mathrm{P} 9.26$

2-Chloro-4-methyl-2-(trichloromethyl)-1,2,3,6-tetrahydropyridine (5). Yield $0.06 \mathrm{~g}$ (3\%), oil. ${ }^{1} \mathrm{H}$ NMR $\left(\mathrm{CDCl}_{3}\right): \delta=2.03(\mathrm{~s}, 3 \mathrm{H}, \mathrm{Me}), 3.34\left(\mathrm{~s}, 2 \mathrm{H}, \mathrm{CCH}_{2}\right), 4.04\left(\mathrm{~d}, 1 \mathrm{H},{ }^{2} J_{\mathrm{HH}}=16 \mathrm{~Hz}\right.$, $\left.\mathrm{NCH}_{\mathrm{A}}\right), 4.72\left(\mathrm{dd}, 1 \mathrm{H},{ }^{2} J_{\mathrm{HH}}=16 \mathrm{~Hz},{ }^{3} J_{\mathrm{HH}}=6 \mathrm{~Hz}, \mathrm{NCH}_{\mathrm{B}}\right), 6.11(\mathrm{~m}, 1 \mathrm{H}, 5-\mathrm{H}) .{ }^{13} \mathrm{C} \mathrm{NMR}\left(\mathrm{CDCl}_{3}\right): \delta$ = $22.53(\mathrm{Me}), 44.76,51.17$ (6-C, 3-C), 90.49, 92.51 (2-C, $\mathrm{CCl}_{3}$ ), 121.04 (5-C), 136.45 (4-C). Anal. Calcd for $\mathrm{C}_{7} \mathrm{H}_{9} \mathrm{Cl}_{4} \mathrm{~N}$ : C 33.77; $\mathrm{H} 3.64 ; \mathrm{Cl} 56.96 ; \mathrm{N} 5.63$. Found: C 33.41; H 3.58; Cl 56.61; N 5.51 .

1-Dichlorophosphoryl-3,4-dimethyl-6-(trifluoromethyl)-1,2-dihydropyridine (7a). A solution of the tetrahydropyridine $3 \mathbf{a}(3.3 \mathrm{~g}, 10 \mathrm{mmol})$ in $3 \mathrm{~mL}$ of trifluoroacetic acid- $\mathrm{d}_{1}$ was stirred for $25 \mathrm{hrs}$, the solvent was evaporated in vacuum at $30-35{ }^{\circ} \mathrm{C}$ to afford oily compound $7 \mathbf{a}$, which was purified by column chromatography (silica gel, hexane/ethyl acetate 10:1). Yield: $1.85 \mathrm{~g}(64 \%) .{ }^{1} \mathrm{H}$ NMR $\left(\mathrm{CDCl}_{3}\right): \delta=1.83$ (s, 3H, Me), 1.87 (s, 3H, Me), 4.09 (d, 2H, ${ }^{3} J_{\mathrm{HP}} 26$ Гц, $\left.\mathrm{NCH}_{2}\right), 6.55(\mathrm{~s}, 1 \mathrm{H}, 5-\mathrm{H}) .{ }^{19} \mathrm{~F} \mathrm{NMR}\left(\mathrm{CDCl}_{3}\right): \delta=-63.2 .{ }^{31} \mathrm{P} \mathrm{NMR}\left(\mathrm{CDCl}_{3}\right): \delta=14.9\left(\mathrm{t},{ }^{3} J_{\mathrm{PH}}=26\right.$ Hz). Anal. Calcd for $\mathrm{C}_{8} \mathrm{H}_{9} \mathrm{Cl}_{2} \mathrm{~F}_{3} \mathrm{NOP}$ : C, 32.68; H, 3.09; Cl, 24.11; N, 4.76; P, 10.53. Found: C, 32.81; H, 3.19; Cl, 23.68; N, 4.61; P, 9.97.

1-Dichlorophosphoryl-4-methyl-6-(trifluoromethyl)-1,2-dihydropyridine (7b) was obtained by thermal distillation of the tetrahydropyridine $\mathbf{3 b}(3.16 \mathrm{~g}, 10 \mathrm{mmol})$ in vacuum $(0.05 \mathrm{mmHg})$. Yield: $1.9 \mathrm{~g}(67 \%)$; bp $\left.118-121^{\circ} \mathrm{C} / 0.05 \mathrm{mmHg} . \%\right) .{ }^{1} \mathrm{H} \mathrm{NMR}\left(\mathrm{CDCl}_{3}\right): \delta=1.80$ (s, 3H, Me), 4.19 $\left(\mathrm{dd},{ }^{3} J_{\mathrm{HP}} 26 \mathrm{~Hz},{ }^{3} J_{\mathrm{HH}} 2.5 \mathrm{~Hz}, \mathrm{NCH}_{2}\right), 5.7(\mathrm{br}, 1 \mathrm{H}, 3-\mathrm{H}), 6.47(\mathrm{~s}, 1 \mathrm{H}, 5-\mathrm{H}) .{ }^{19} \mathrm{~F} \mathrm{NMR}\left(\mathrm{CDCl}_{3}\right): \delta=$ -63.3. ${ }^{31} \mathrm{P}$ NMR $\left(\mathrm{CDCl}_{3}\right): \delta=14.1\left(\mathrm{t},{ }^{3} J_{\mathrm{PH}}=26 \mathrm{~Hz}\right)$. Anal. Calcd for $\mathrm{C}_{7} \mathrm{H}_{7} \mathrm{Cl}_{2} \mathrm{~F}_{3} \mathrm{NOP}: \mathrm{C} 30.03 ; \mathrm{H}$ 2.52; Cl 25.32; N 5.00; P 11.06. Found: C 29.83; H 2.49; Cl 25.91; N 4.87; P 10.84.

\section{General procedures for the synthesis of pyridines (8) and (9)}

The mixture of respective tetrahydropyridine 3 or dihydropyridine 7 (10 mmol), dioxane $(15 \mathrm{~mL})$ and $35 \%$ aq $\mathrm{HCl}(15 \mathrm{~mL})$ was refluxed for $3 \mathrm{hrs}$. After cooling to r.t. the mixture was neutralized with $10 \% \mathrm{NaOH}$, extracted with dichloromethane $(2 \times 25 \mathrm{~mL})$, dried $\left(\mathrm{MgSO}_{4}\right)$ and the solvent was removed in vacuum at $50-60{ }^{\circ} \mathrm{C}$. 
4,5-Dimethyl-2-(trifluoromethyl)pyridine (8a). Yield 63\% (from 3a) or 66\% (from 7b); bp 75$80{ }^{\circ} \mathrm{C}(14 \mathrm{~mm})\left(\right.$ lit. ${ }^{15} \mathrm{bp} 85{ }^{\circ} \mathrm{C}(17 \mathrm{~mm}){ }^{1} \mathrm{H} \mathrm{NMR}\left(\mathrm{CDCl}_{3}\right): \delta=2.29$ (s, 3H, Me), $2.33(\mathrm{~s}, 3 \mathrm{H}$, $\mathrm{Me}), 7.41$ (s, 1H, 3-H), 8.40 (s, 1H, 6-H). ${ }^{19} \mathrm{~F} \mathrm{NMR}\left(\mathrm{CDCl}_{3}\right): \delta=-67.40$.

4-Methyl-2-(trifluoromethyl)pyridine (8b). Yield: $81 \%$ (from 3b); $n_{\mathrm{D}}{ }^{20} 1.4292$ (lit. $^{16} n_{\mathrm{D}}{ }^{20}$ 1.429). ${ }^{1} \mathrm{H}$ NMR $\left(\mathrm{CDCl}_{3}\right): \delta=2.44(\mathrm{~s}, 3 \mathrm{H}, \mathrm{Me}), 7.27$ (d, $\left.1 \mathrm{H},{ }^{3} J_{\mathrm{HH}}=5 \mathrm{~Hz}, 5-\mathrm{H}\right), 7.49$ (s, $1 \mathrm{H}, 3-$ $\mathrm{H}), 8.56\left(\mathrm{~d}, 1 \mathrm{H},{ }^{3} J_{\mathrm{HH}}=5 \mathrm{~Hz}, 6-\mathrm{H}\right) .{ }^{19} \mathrm{~F} \mathrm{NMR}\left(\mathrm{CDCl}_{3}\right): \delta=-68.77 . \mathrm{m} / \mathrm{z}(\mathrm{APCI}) 162.2(\mathrm{M}+1$, $100 \%)$. Calculated for $\mathrm{C}_{7} \mathrm{H}_{6} \mathrm{~F}_{3} \mathrm{~N}, \mathrm{M}=161.13$.

Compound $\mathbf{8 b}$ was prepared also by the following procedure: $3.17 \mathrm{~g}(10 \mathrm{~mol})$ of tetrahydropyridine $\mathbf{3 b}$ was distilled, fraction with bp 170-180 ${ }^{\circ} \mathrm{C}$ was collected, dissolved in dichloromethane $(50 \mathrm{~mL})$, washed with $10 \% \mathrm{NaOH}$ and dried $\left(\mathrm{MgSO}_{4}\right)$. The solvent was removed in vacuum at $50-60{ }^{\circ} \mathrm{C}$ to afford $1.1 \mathrm{~g}(68 \%)$ of pyridine $\mathbf{8 b}$.

2-(Dichloromethyl)-4,5-dimethylpyridine (9a). Yield: $1.2 \mathrm{~g}(63 \%)$; bp $115-120{ }^{\circ} \mathrm{C}(14 \mathrm{~mm})$; mp 70-72 ${ }^{\circ} \mathrm{C}$ (hexane-benzene 3:1). ${ }^{1} \mathrm{H}$ NMR $\left(\mathrm{CDCl}_{3}\right): \delta=2.19$ (s, 3H, Me), 2.25 (s, 3H, Me), $6.63\left(\mathrm{~s}, 1 \mathrm{H}, \mathrm{CHCl}_{2}\right), 7.45$ (s, 1H, 3-H), 8.19 (s, 1H, 6-H). ${ }^{13} \mathrm{C} \mathrm{NMR}\left(\mathrm{CDCl}_{3}\right): \delta=16.28(\mathrm{Me})$, 19.43 (Me), $71.48\left(\mathrm{CHCl}_{2}\right), 121.77$ (3-C), 133.61 (5-C), 147.71 (4-C), 148.74 (6-C), 155.50 (2C). Anal. Calcd for $\mathrm{C}_{8} \mathrm{H}_{9} \mathrm{Cl}_{2} \mathrm{~N}$ : C 50.55; $\mathrm{H} 4.77 ; \mathrm{Cl} 37.30 ; \mathrm{N}$ 7.37. Found: $\mathrm{C} 49.97 ; \mathrm{H} \mathrm{4.68;} \mathrm{Cl}$ 37.26; N 7.41 .

2-(Dichloromethyl)-4-methylpyridine (9b). Yield: $1.1 \mathrm{~g}(68 \%)$; bp 160-170 ${ }^{\circ} \mathrm{C}$ (lit. ${ }^{17}$ bp 74-75 ${ }^{\circ} \mathrm{C}(14 \mathrm{~mm}) ;{ }^{1} \mathrm{H}$ NMR $\left(\mathrm{CDCl}_{3}\right): \delta=2.43(\mathrm{~s}, 3 \mathrm{H}, \mathrm{Me}), 6.71\left(\mathrm{~s}, 1 \mathrm{H}, \mathrm{CHCl}_{2}\right), 7.14\left(\mathrm{~d}, 1 \mathrm{H},{ }^{3} J_{\mathrm{HH}}=5\right.$ $\mathrm{Hz}, 5-\mathrm{H}), 7.58(\mathrm{~s}, 1 \mathrm{H}, 3-\mathrm{H}), 8.43\left(\mathrm{~d}, 1 \mathrm{H},{ }^{3} J_{\mathrm{HH}}=5 \mathrm{~Hz}, 6-\mathrm{H}\right) .{ }^{13} \mathrm{C} \mathrm{NMR}\left(\mathrm{CDCl}_{3}\right): \delta=21.12(\mathrm{Me})$, $71.59\left(\mathrm{CHCl}_{2}\right), 121.73,125.38$ (3-C, 5-C), 148.50, 149.13 (4-C , 6-C), 157.68 (2-C).

\section{References}

1. (a) Petrova, T. D.; Platonov, V. E. Usp. Khim. 1988, 57, 405; Russ. Chem. Revs., 1988. (b) Ulrich, T. The Chemistry of Imidoyl Halides. N.Y.: Plenum Press, 1968. (c) Trost, B. M. In Comprehensive Organic Synthesis; Winterfeldt, E.; Ed.; Pergamon Press: N. Y, 1991; Vol. 6, p 523. (d) Alonso, E.; Ramon, D. J.; Yus, M. Tetrahedron, 1998 , 54, 12007. (e) Onys'ko, P. P.; Rassukana, Y. V.; Sinitsa A. D. J. Org. Pharm. Chem. (Ukraine). 2009, 7, 37. (f) Chen, M. W.; Zhang, X. G.; Zhong, P.; Hu, M. L. Synthesis 2009, 1431.

2. (a) Rassukana, Y. V.; Onys'ko, P. P.; Grechukha A. G.; Sinitsa A. D. Eur. J. Org. Chem. 2003, 21, 4181. (b) Rassukana, Y. V.; Khomenko, E. A.; Onys'ko, P. P.; Sinitsa, A. D. Synthesis 2006, 19, 3195. (c) Onys'ko, P. P.; Rassukana, Y. V.; Sinitsa, O.A. Phosphorus, Sulfur, Silicon and the Related Elements 2008, 183, 399. (d) Wang, Z.; Ge, F.; Wan, W.; Jiang, H.; Hao, J. J. Fluor. Chem. 2007, 128, 1143. Uneyama, K. J. Fluor. Chem. 1999, 97, 11.

3. Smits, R; Cadicamo, C. D.; Burger, K; Koksh, B. Chem. Soc. Rev. 2008, 37, 1727.

4. Organofluorine Compounds in Medicinal Chemistry and Biomedicinal Applications; Filler, R.; Kobayashi, Y.; Yagupolskii, L. M., Eds.; Elsevier; Amsterdam, 1993. 
5. Chupp, J. P.; Smith, L. R. J. Heterocyclic Chem. 1988, 25, 1785.

6. (a) Chupp, J. P.; J. Heterocyclic Chem. 1990, 27, 1697. (b) Lee, L. F.; Miller, M. L.; Sing, Y. L. EP 278 945, 1988; Chem. Abstr. 1989, 110, 57521q.

7. Roesky, H. W.; Giere, H. H.; Z. Anorg. Alg. Chem. 1970, 78, 177.

8. Exner, O. In Correlation Analysis in Chemistry; Chapman, N. B.; Shorter, J. Eds.; Plenum Press: N. Y, 1978; p 439.

9. Polozov, A.M.; Mustaphin, A. H.; Khotinen, A.V. Phosphorus, Sulfur, Silicon 1992, 73, 153.

10. Kolotylo, M. V.; Shalimov, O. O.; Onys'ko, P. P.; Sinitsa A. D., Ukrainian Patent, 59199, 2011.

11. Onys'ko, P. P.; Rassukana, Y. V.; Sinitsa, O. A. Curr. Org. Chem. 2010, 14, 1223.

12. Soloshonok V.A.; Ono, T. Tetrahedron 1996, 52, 14701.

13. Derkach, G. I.; Rudavskii, V. P.; Koval, A. A.; Shevchenko, V. I. J. Gen. Chem. USSR (Engl. Transl.) 1967, 37, 415.

14. Shevchenko, V. I.; Nizhnikova, E. E.; Bodnarchuk, N. D.; Kornuta, P. P. J. Gen. Chem. USSR (Engl. Transl.) 1967, 37, 1284.

15. Vasil'ev, N. V.; Romanov, D. V.; Lyssenko, K. A.; Zatonsky, G. V. Fluor. Chem. 2007, $128,740$.

16. Janz, G. J.; Monahan, A. R.; J. Org. Chem. 1965, 30, 1949.

17. Katagiri, N.; Niwa, R.; Fuzuya, T.; Kato, T. Chem. Pharm. Bull. 1983, 31, 1833. 